\title{
A systematic Review Study on Educational Interventions to Reduce Nurses Job Stress
}

\author{
Mostafa Madmoli1 ${ }^{*}$, Mohammad Madmoli2, Mohaddeseh Izadi², Mahboobeh Khodadadi3, \\ Fahimeh Papi Ahmadi ${ }^{3}$
}

${ }^{1}$ Emergency Medical Technician, Dezful University of Medical Sciences, Dezful, Iran*.

${ }^{2}$ Student Research Committee Shoushtar faculty of Medical Sciences, Shoushtar, Iran.

${ }^{3}$ Nursing Student, Student Research Committee, Dezful University of Medical Sciences, Dezful, Iran.

\begin{abstract}
:
Introduction: Excessive stress impairs performance and reduces the productivity of employees in organizations. Therefore, stress is one of the issues that is being explored in the field of management and organizational behavior. Therefore, organizations in their program have a lot of attention to studying, controlling and reducing stress in the workplace, in order to reduce the cost of physical and psychological damage to stress. And it also boosts the productivity of its employees. Stress management can be very important in using educational programs. This systematic review was conducted with the aim of determining educational interventions in reducing nursing job stress.G
\end{abstract}

Materials and Methods: In this systematic review using articles published in the last 25 years about educational interventions to reduce nursing job stress. It was conducted in English and Persian by searching articles in search engines, sites and authoritative scientific databases SID, Magiran, Google Scholar, Embase, Researchgate, ScienceDirect, PubMed, Springer. In the first stage, 35 papers were found. Of these, 10 related articles that have been published in the last 25 years have been reviewed.G

Results: In this study, ten articles have reviewed that show the positive effect of training on reducing the stress of nurses. One of these studies was to investigate the effect of critical thinking training on occupational stress on nurses. Revealed a significant reduction in the job stress score among the subjects of the critical thinking training group and These results were also significant in comparison with the control group.G

Conclusion: Many workplaces suffer from work stress in workplaces but they are not aware of the proper solution to prevent and manage this stress. It can be said that the implementation of vocational stress management training programs by increasing the level of employees' awareness of how to manage occupational stress, how to better manage time and also to modify work interactions to reduce job stress, this problem can be reduced among employees. And it can improve your job satisfaction day by day.

Keywords: Educational interventions, Job stress, Nurses

\section{Introduction}

Neuropsychiatric pressure is considered by physicians, psychologists, and behavioral science scientists as the most important factor in the physical, psychological and behavioral disorders of humans. The importance of stress is not only due to its economic damage, but also its negative effects on the physical and mental health of individuals. Today, it has been determined that stress is associated with many physical and mental illnesses, or contributes to its development, development, and development (1-3).

21 International Journal of Drug Research and Dental Science 
Social and psychological factors not only affect the quality of life but also often play a role in determining the outcome of coping with a chronic disease (4). Stress along with many illnesses will cause many complications, for example, stress with diabetes will increase blood sugar and further complications of diabetes. Diabetes, a chronic and metabolic disease (5-12), has always been interacting with stress. Complications of diabetes are very common among people with this condition and they will be heavily stressed (12-20).G

At the moment, stress and depression are the problems of youth and adolescents. Job stress is a very common feature of modern life and is increasing and has a bad effect on cardiovascular systems. Today it is known that stress is associated with many physical and mental illnesses or contributes to its development, development, and development. Another dimension that makes people interested in studying stress is the relationship between stress and performance (21-29). Excessive stress impairs performance and reduces the productivity of employees in organizations. Therefore, stress is one of the issues that is being explored in the field of management and organizational behavior. Therefore, organizations in their program pay much attention to the study, control, and reduction of stress in the work environment, in order to reduce the cost of physical and psychological injuries and also increase the productivity of their employees (30). Stress management can be very important in using educational programs. This systematic review was conducted with the aim of determining educational interventions in reducing nursing job stress.G

\section{Materials and Methods}

In order to achieve the goal of the study and to improve the accuracy of its study and its comprehension, this integrated overview study was conducted based on the Broome method. The method is done in the form of three steps in the search of texts, data evaluation, and data analysis, So, in the search phase, post-retention studies texts are examined in four stages in terms of inclusion criteria. After obtaining the conditions for entry into the study, the content of the study is evaluated and at the end, the analysis of the data was done.G In this systematic review using articles published in the last 25 years about educational interventions to reduce nursing job stress. It was conducted in English and Persian by searching articles in search engines, sites and authoritative scientific databases SID, Magiran, Google Scholar, Embase, Researchgate, ScienceDirect, PubMed, Springer. In the first stage, 35 papers were found. Of these, 10 related articles that have been published in the last 25 years have been reviewed.G

The criteria for entry of articles include studies published in Persian and in English, Access to their full text was possible, they were published in the last 25 years and Exit criteria include unnamed and non-scientific studies as well as articles that lacked the full text of the article.

To achieve relevant studies, a wide range of keywords including Educational interventions, Job stress, and Nurses was used as a one-to-one search, combined with the method "And" and "OR".G

Results: The results of the study on the effect of stress management training program on stress and worklife conflict showed that the stress level in the intervention group was significantly lower than the control group. Also, in the intervention group itself, the work stress was reduced compared to before the intervention. The results of the intervention showed that, by reducing work stress, the work-life conflict scores also decreased and there is a significant positive correlation between stress management and worklife conflicts in nurses (31).

Another study (32) found that there was a positive correlation between work stress and work-life conflict in investigating the relationship between work stress and work-life conflict.

Another study quoted (33), aimed at determining the effect of stress relief program on perceived stress by nurses, showed that training in the test group significantly decreases the stress score but there is no difference in the control group. Also, comparing the scores of both groups before and after the intervention showed a significant difference after the intervention, indicating a decrease in the stress level of the subjects

22 International Journal of Drug Research and Dental Science 
in the intervention group. This indicates the effect of stress-relief immunization program on the stress of nurses working in the mental health sector.

Another study showed that the average stress of legal students who received four weeks of stress relief training received $6.6 \%$ of the post-intervention phase and $4.6 \%$ at the follow-up stage (34).

In another study, there was a significant difference between the mean of work-life conflict before and one month after the intervention in the experimental group. Also, there was a significant difference in the mean of stress before and one month after the intervention in the experimental group. There was also a significant difference between the mean of work stress and work-life conflict before and after intervention and intervention in the two groups (35).

In another study, the effect of the educational program on functional relaxation based on the PRESSED model was studied on the reduction of nurses' anxiety. The intervention design was based on the PRECEDE model that for the predisposing and enabling factors of the classes, three classes were segregated for 7 weeks, each week, one day and every day, between one and one and a half hours. And applied relaxation techniques were considered as objective behaviors that using short-term lectures with questions and answers, group discussions, practice shows, and pamphlets were taught. After the end of the 7 training sessions, the recall was carried out through a short message of up to one month and an instructor's attendance. Findings of the study after 6 months showed a significant difference between the mean score of the apparent and hidden anxiety in the intervention group compared to the pre-intervention group (36).G

The study to investigate the effect of critical thinking training on job stress on nurses revealed a significant reduction in the job stress score among students of critical thinking education group and these results were also significant in comparison with the control group (37).

In another study quoted in a study (33), in order to investigate the effect of a focus-based training program and deep breathing exercises to reduce the symptoms of stress on the nurse, the novelty of this study was to measure serum cortisol levels which were examined before and after an intervention. The results showed a positive effect of training on the level of work-related stress in the test group and the comparison of the scores in the test group was significant compared to the control group.G

In a study (38), the effect of training based on the stress-relief model on emergency department and trauma nurses was done. In this study, the stability model was used against stress conditions and four areas of occupational safety and health and care in the mental and environmental conditions that were also observed in the training. The results indicated a reduction in the stress score due to exposure to stressed materials and stressful conditions in the test group. Also, the knowledge and awareness of the nurses in the intervention group were increased in advance and the results of this study coincided with similar studies in the field of education, occupational stress and the nature of nursing to reduce stress $(39,40) . G$

Discussion: Some researchers have focused on stress-related work stressors in these high-profile jobs, and some on the individual's individual situation. Considering the relatively high prevalence of stress and since the initial failure, early diagnosis and timely control of this disorder, initiates and persists, the use of preventive methods includes training methods in recognizing stress in the early stages and coping with it is important (43). Stress management training programs increase individuals' ability to reduce stress and adapt to stressful situations (44).G

Therefore, the use of appropriate instructional theory (as the basis of educational work) helps these people to recognize symptoms of stress and to deal with the first signs of stress correctly (45). According to study (31), we can say the application of stress management skills in work and family life by nurse's better time management, work and individual stress, proper dieting, Correct way of working in the family and working environment helps to balance work and life.G 
According to the results of another study quoted in (33), which aimed to determine the effect of the program of immunization against stress on nurses' perceived stress that the training in the test group significantly reduces the stress score, it can be said that since the stress management program is a cheap, effective and safe way to reduce the perceived stress of nurses, therefore, it is recommended to use nurses' effective coping with job stress and improve the quality of care.

According to the results of the study (36), it can be said that the findings confirm the effect of an educational program based on the PRECEDE pattern to reduce the anxiety of nurses. It seems that the use of educational interventions based on the PRECEDE model, especially physical and mental health education, can help to improve the health of the target community in specific communities and the community as a whole.G

Conclusion: Many workplaces suffer from work stress in workplaces but they are not aware of the proper solution to prevent and manage this stress. It can be said that the implementation of vocational stress management training programs by increasing the level of employees' awareness of how to manage occupational stress, how to better manage time and also to modify work interactions to reduce job stress, this problem can be reduced among employees. And it can improve your job satisfaction day by day.

\section{Acknowledgment}

The authors of this article are grateful to all those who have collaborated with me in this article.G

\section{References}

1. vosoughi niri A, ruhollahi A, mohmmad hosein H. A survey of the effect of job stress on general health and job performance on Air Traffic Controllers (ATC). ioh. 2016; 13 (1):47-57G

2. Khosravi M. Examine job stressors librarians working in academic libraries Ministry of Science, Research and Technology, Tehran, from their perspective, research projects, Information, and Documentation Center of Iran, Tehran, 2004.

3. Rhodes J, Smith B. Combat stress. Marine Corps Reference, 2000:p.16-40.

4. AMIRI M, AGHAEI A, ABEDI A, SAFARI Y. THE EFFECT OF STRESS INOCULATION TRAINING ON DEPRESSIVE SYMPTOMS DIABETES. 2014; 16-30.

5. Moslemirad M, Madmoli M, Madmoli Y, Niksefat M. Prevalence of type 1 and type 2 diabetes and its related factors in diabetic patients hospitalized in Khatam-ol-Anbia hospital in Shoushtar, 2014-15: A retrospective study. Journal of Research in Medical and Dental Science. 2018;6(3):421-6

6. Madmoli M, Eilami O, Rezaie K, Aliabad MA, Moslemirad M. Diabetes and the risk of suffering cardiovascular Diseases: A two-year retrospective study. International Journal of Ecosystems and Ecology Science (IJEES). 2018 Jun;8(3): 649-56.

7. Madmoli M Rostami F, Mirsami Yazdi N, Mosavi A, Baraz Sh. Evaluation of Prevalence of Diabetic Foot Ulcer and Its Related Factors in Diabetic Patients Admitted to KHatam-ol-Anbia Hospital in Shoushtar During 2015-2016: A Retrospective Study. International Journal of Ecosystems and Ecology Science (IJEES). 2018 June; 8 (3): 545-52.

8. Rostami F, Madmoli M, Mirsami Yazdi N, Baraz Sh. Evaluation of The Prevalence of Lower Limb Amputation and Its Related Factors in Diabetic Patients Admitted to KHatam-ol-Anbia Hospital in Shoushtar During The 2015-2016: A Retrospective Study. International Journal of Ecosystems and Ecology Science (IJEES). 2018 June; 8 (3): 553-60.

9. Raisifar Z, Afshar Nia A, Madmoli M, Madmoli Y. The Relationship Between Using Insulin and Suffering Alzheimer's Disease in Patients with Diabetes: A Two-Year Study. International Journal of Ecosystems and Ecology Science (IJEES). 2018 June; 8 (3): 623-28.

10. Raisifar Z, Afshar Nia A, Maghamesi Moarrefi H, Madmoli M. Evaluation of Gi Bleeding Prevalence and Its Related Factors in Diabetic Patients Hospitalized in KHatam-ol-Anbia Hospital During 2015-16: A Retrospective Study. International Journal of Ecosystems and Ecology Science (IJEES). 2018 June; 8 (3): 609-14. 
11. Madmoli M, Kord Z, Bandani A, Sedighi N, Rezaei Shandiz M, Darabiyan P, AfsharNia A. Epidemiological and clinical study of patients with Alzheimer's in Five Cities of Khuzestan Province in 2016-2018. Medical Science, 2019; 23(95), 1-5

12. Madmoli M, Fallah bagher shaidaei M, Rohani A, Darabiyan P, Mobarez F. The correlation between alcohol consumption and reducing the age of cancer incidence in patients with this disease. Medical Science, 2019, 23(95), 48-53

13. Madmoli M, Mahmoudi Dehcheshmeh Z, Rafi A, Zahra Kord, Fariba Mobarez, Pouriya Darabiyan. The rate of some complications and risk factors of diabetes in diabetic patients: Study on cases of 3218 diabetic patients. Medical Science, 2019; 23(95), 63-68

14. Mostafa Madmoli, Mehran Yarbig, Negin Sedighi, Pouriya Darabiyan, Fariba Mobarez. Communication between body mass index and the risk of obesity-related cancer: A 5-year study on patients with cancer. Medical Science, 2019; 23(95), 69-74

15. Madmoli M, Madmoli Y, Khodadadi M, Samsamipour M. Some Factors Affecting Quality of Life in Patients with Diabetes: A Systematic Review. Annals of Microbiology and Infectious Diseases. 2019; 2(1). 26-30.

16. Madmoli M. Clinical and Laboratory Finding Children with Leukemia: a Systematic Review. International Journal of Research Studies in Science, Engineering, and Technology, vol. 5, no. 10, pp. 2018; $1-6$

17. Madmoli M. Evaluation of Chemotherapy Complications in Patients with Cancer: A Systematic Review. International Journal of Research Studies in Science, Engineering, and Technology, vol. 5, no. 12, 2018; 59-64.G

18. Madmoli M. Quality of Life in Patients with Cancer and Some Factors Affecting it: A Systematic Review. International Journal of Research Studies in Science, Engineering, and Technology. 6(1), 2019; 1-7G

19. Madmoli M, Madmoli Y, Khodadadi M, Samsamipour M. Study of Some Effective Treatments for Accelerating Diabetic Foot Ulcer Healing: A Systematic Review. International Journal of Research Studies in Science, Engineering and Technology, 6(2), 2019; 34-39

20. Madmoli M, Madmoli Y, Khodadadi M, Samsamipour M. Factors Affecting the Level of Glycosylated Hemoglobin in Patients with Diabetes: A Systematic Review. Annals of Microbiology and Infectious Diseases 2(1), 2019; 43-47.

21. Gheisari Z, Beiranvand R, Karimi A, Ghalavandi S, Soleymani A, Madmoli M, Bavarsad AH. Relationship between Occupational Stress and Cardiovascular Risk Factors Determination: A Casecontrol Study. Journal of Research in Medicaland Dental Science. 2018 May 17;6(3):287-93.

22. Madmoli M, Nikpay S. An Investigation of the Relationship between Spiritual Health and Depression, Anxiety, and Stress among Students of Ilam University of Medical Sciences. Journal of Research in Medical and Dental Science. 2018 May 17;6(3):294-300.

23. Madmoli Y, Akhaghi Dezfuli SM, Beiranvand R, Saberi Pour B, Azami M, Madmoli M.

24. Mashali H, Toleideh F, Rahmani R, Darabiyan P, Madmoli M. The predictive role of Hyperlipidemia in the incidence of ACS in patients referring to Shahidzadeh Hospital in Behbahan in 2016 -2017. Medical Science, 2018; 22(94), 566-570

25. Madmoli Y, Madmoli M, Qashqaei Nezhad N, Bosak S. Prevalence of depression and associated factors in adolescents of Masjed-Soleyman. Iranian Journal of Pediatric Nursing. 2016.

26. Roughani A, Madmoli M, Raisifar Z, Kikhavani S, Yasemi M, Azami M, Sharifi Z. The prevalence of behavioral disorders and its related factors in elementary school children in Ilam City in 2011-2012. Indian Journal of Forensic Medicine \& Toxicology. 2018;12(4):165-9.

27. Adavi A, Hamid N, Attari Y, Madmoli Y, Madmoli M. Study of the Effect of Problem-Solving Skills Training on Creativity and Assertiveness Among High School Students. Iranian Journal of Nursing Research. 2016 Dec 15;11(5):48-55. 
28. Madmoli M, Madmoli Y, Bigdeli Shamloo MB, Etebari A, Mahmoodi Kouhi A, Azami M. The Relationship Between Depression and Religiousness in Female High School Students of Masjed Soleyman in 2015. Journal of Pediatric Nursing. 2017 Jun 15;3(4):15-22.

29. Madmoli Y, Aslani A, Ahmadi Y, Mousavi M, Mashalchi H, Niksefat M, Madmoli M. Study Habits and Related Factors in Students of Nursing and Midwifery College of Dezful University of Medical Sciences in 2015. Iranian Journal of Nursing Research (IJNR). 2017.

30. VOSOUGHI NA, ROHOLLAHI A, MOHAMAD HH. The effect of job stress on general health and job performance on air traffic controllers (etc).2016; 47-57.G

31. Makie VV. Stress and coping strategies amongst registered nurses working in a South African tertiary hospital: University of the Western Cape; 2006.

32. Chuang Y, Lei Y. Retail staffs working stress and work-family conflict under shifts: Work system study. BAI Conference Papers2011.

33. Hazavehei SM, Moghadam K, Melika S, Bagheri Kholenjani F, Ebrahimi H. The influence of educational interventions to reduce occupational stress: A systematic review. Health and Safety at Work. 2017 Dec 15;7(4):363-74.

34. Sheehy R, Horan JJ. Effects of Stress Inoculation Training for 1st-Year Law Students. International Journal of Stress Management. 2004;11(1):41.

35. Alavi Arjmand N, Kashaninia Z, Hosseini MA, Rezasoltani P. Effect of stress management on job stress and work-family conflict among nurses. Journal of hayat. 2013;18(4):81-91.

36. Ebrahimi Iraqi Nezhad Z, Tol A, Shojaeezadeh D, Khorsandi M, Bagheri F. Effectiveness of PRECEDE model for health education on anxiety of nurses employed in hospitals of Arak University of Medical Sciences: application of PRECEDE model constructs Anxiety of nurses and PRECEDE model. J Health Syst Res. 2014;10(4):752-65.

37. Vaghee S, Meshkin Ya, Asgharipoor N, Ebrahimzadeh S. The Effect of Critical Thinking Training On Nurses'job Stress in Psychiatric Ward. 2014.

38. Moayed MS, Mahmoudi H, Ebadi A, Salary MM, Danial Z. Effect of Education on Stress of Exposure to Sharps Among Nurses in Emergency and Trauma Care Wards. Trauma monthly. 2015;20(2).

39. Gelsema TI, Van Der Doef M, Maes S, Akerboom S, Verhoeven C. Job Stress in the Nursing Profession: The Influence of Organizational and Environmental Conditions and Job Characteristics. International Journal of Stress Management. 2005;12(3):222.

40. Delvaux N, Razavi D, Marchal S, Brédart A, Farvacques C, Slachmuylder J-L. Effects of a 105 hours psychological training program on attitudes, communication skills and occupational stress in oncology: a randomized study. British journal of cancer. 2004;90(1):106-14.G

41. Phillips MR, Zhang J, Shi Q, Song Z, Ding Z, Pang S, et al. Prevalence, treatment, and associated disability of mental disorders in four provinces in China during 2001-05: an epidemiological survey. The Lancet. 2009;373(9680):2041-53.

42. Kawano Y. Association of job-related stress factors with psychological and somatic symptoms among Japanese hospital nurses: effect of departmental environment in acute care hospitals. Journal of occupational health. 2008;50(1):79-85.

43. Jones SC, Donovan RJ. Does theory inform practice in health promotion in Australia? Health Education Research. 2004;19(1):1-14.

How to cite this Article: Mostafa Madmoli, Mohammad Madmoli, Mohaddeseh Izadi, Mahboobeh Khodadadi, Fahimeh Papi Ahmadi, A systematic Review Study on Educational Interventions to Reduce Nurses Job Stress, Int. J. Drug Res. Dental Sci., 2019; 1(1):21-26.

Source of Support: Nil, Conflict of Interest: None declared. 